\title{
Measurement of Tensor Polarization in Elastic Electron-Deuteron Scattering at Large Momentum Transfer
}

\author{
D. Abbott, ${ }^{4}$ A. Ahmidouch,,${ }^{6,10}$ H. Anklin, ${ }^{11}$ J. Arvieux, ${ }^{9,12}$ J. Ball, ${ }^{2,9}$ S. Beedoe, ${ }^{10}$ E. J. Beise, ${ }^{3}$ L. Bimbot, ${ }^{12}$
}

W. Boeglin, ${ }^{11}$ H. Breuer, ${ }^{3}$ P. Brindza, ${ }^{4}$ R. Carlini, ${ }^{4}$ N. S. Chant, ${ }^{3}$ S. Danagoulian, ${ }^{10,4}$ K. Dow, ${ }^{6}$ J.-E. Ducret, ${ }^{2}$ J. Dunne, ${ }^{4}$ L. Ewell, ${ }^{3}$ L. Eyraud, ${ }^{1}$ C. Furget, ${ }^{1}$ M. Garçon, ${ }^{2}$ R. Gilman, ${ }^{4,7}$ C. Glashausser, ${ }^{7}$ P. Gueye,${ }^{4}$ K. Gustafsson, ${ }^{3}$ K. Hafidi, ${ }^{2}$ A. Honegger ${ }^{8}$ J. Jourdan, ${ }^{8}$ S. Kox,${ }^{1}$ G. Kumbartzki, ${ }^{7}$ L. Lu, ${ }^{1}$ A. Lung, ${ }^{3}$ D. Mack, ${ }^{4}$ P. Markowitz, ${ }^{11}$ J. McIntyre, ${ }^{7}$ D. Meekins, ${ }^{4}$ F. Merchez, ${ }^{1}$ J. Mitchell, ${ }^{4}$ R. Mohring, ${ }^{3}$ S. Mtingwa, ${ }^{10}$ H. Mrktchyan, ${ }^{13}$ D. Pitz, ${ }^{2,3,4}$ L. Qin, ${ }^{4}$

R. D. Ransome, ${ }^{7}$ J.-S. Réal, ${ }^{1}$ P. G. Roos, ${ }^{3}$ P. Rutt, ${ }^{7}$ R. Sawafta, ${ }^{10,4}$ S. Stepanyan, ${ }^{13}$ R. Tieulent, ${ }^{1}$ E. Tomasi-Gustafsson, ${ }^{2,9}$ W. Turchinetz, ${ }^{5}$ K. Vansyoc, ${ }^{4}$ J. Volmer, ${ }^{4}$ E. Voutier, ${ }^{1}$ W. Vulcan, ${ }^{4}$ C. Williamson, ${ }^{5}$ S. A. Wood, ${ }^{4}$ C. Yan, ${ }^{4}$

J. Zhao, ${ }^{8}$ and W. Zhao ${ }^{5}$

(The Jefferson Lab t20 Collaboration)

${ }^{1}$ Institut des Sciences Nucléaires, IN2P3-UJF, F-38026, Grenoble, France

${ }^{2}$ DAPNIA/SPhN, CEA/Saclay, F-91191 Gif-sur-Yvette, France

${ }^{3}$ University of Maryland, College Park, Maryland 20742

${ }^{4}$ Thomas Jefferson National Accelerator Facility, Newport News, Virginia 23606

${ }^{5}$ M.I.T.-Laboratory for Nuclear Science and Department of Physics, Cambridge, Massachusetts 02139

${ }^{6}$ M.I.T.-Bates Linear Accelerator, Middleton, Massachusetts 01949

${ }^{7}$ Rutgers University, Piscataway, New Jersey 08855

${ }^{8}$ Basel Institut für Physik, Basel, Switzerland

${ }^{9}$ LNS-Saclay, F-91191 Gif-sur-Yvette, France

${ }^{10}$ North Carolina A. \& T. State University, Greensboro, North Carolina 27411

${ }^{11}$ Florida International University, Miami, Florida 33199

${ }^{12} I P N O, I N 2 P 3, B P 1, F-91406$ Orsay, France

${ }^{13}$ Yerevan Physics Institute, 375036 Yerevan, Armenia

(Received 24 January 2000)

\begin{abstract}
Tensor polarization observables $\left(t_{20}, t_{21}\right.$, and $\left.t_{22}\right)$ have been measured in elastic electron-deuteron scattering for six values of momentum transfer between 0.66 and $1.7(\mathrm{GeV} / c)^{2}$. The experiment was performed at the Jefferson Laboratory in Hall C using the electron High Momentum Spectrometer, a specially designed deuteron magnetic channel and the recoil deuteron polarimeter POLDER. The new data determine to much larger $Q^{2}$ the deuteron charge form factors $G_{C}$ and $G_{Q}$. They are in good agreement with relativistic calculations and disagree with perturbative QCD predictions.
\end{abstract}

PACS numbers: 25.30.Bf, 13.40.Gp, 21.45.+v, 24.70.+s

The development of a quantitative understanding of the structure of the deuteron, the only two-nucleon bound state, has long been considered an important testing ground for models of the nucleon-nucleon potential. Nevertheless, the charge distribution of the deuteron is not well known experimentally, because it is only through the use of both polarization measurements and unpolarized elastic scattering cross sections that it can be unambiguously determined. In the experiment described here, a precise determination of the charge form factor of the deuteron is presented through measurement of the deuteron tensor polarization observables up to a momentum transfer of $Q^{2}=1.7(\mathrm{GeV} / c)^{2}$, for the first time well beyond its zero crossing.

Since the deuteron is a spin-1 nucleus, its electromagnetic structure is described by three form factors: the charge monopole $G_{C}$, the quadrupole $G_{Q}$, and the magnetic dipole $G_{M}$. Thus it is possible to unambiguously separate the three components only through measurement of three observables. In the one-photon exchange approxi- mation, the elastic scattering cross section is typically expressed in terms of structure functions $A\left(Q^{2}\right)$ and $B\left(Q^{2}\right)$ $\left[d \sigma / d \Omega \propto S\right.$ with $S=A\left(Q^{2}\right)+B\left(Q^{2}\right) \tan ^{2}\left(\theta_{e} / 2\right)$; see full expressions, e.g., in [1]] that can be separately determined by variation of the scattered electron angle $\theta_{e}$ for a given momentum transfer $Q^{2}$ to the deuteron.

The third observable can be the cross section dependence on deuteron (tensor or vector) polarization. The tensor analyzing powers can be measured using a polarized deuteron target (with unpolarized beam) [2-5]. Alternatively, the tensor moments of the outgoing deuterons can be measured using unpolarized beam and target [6,7]. Both types of experiment result in the same combinations of form factors:

$$
\begin{aligned}
t_{20}= & -\frac{1}{\sqrt{2} S}\left(\frac{8}{3} \eta G_{C} G_{Q}+\frac{8}{9} \eta^{2} G_{Q}^{2}\right. \\
& \left.+\frac{1}{3} \eta\left[1+2(1+\eta) \tan ^{2} \frac{\theta_{e}}{2}\right] G_{M}^{2}\right),
\end{aligned}
$$




$$
\begin{gathered}
t_{21}=\frac{2}{\sqrt{3} S \cos \frac{\theta_{e}}{2}} \eta\left[\eta+\eta^{2} \sin ^{2} \frac{\theta_{e}}{2}\right]^{1 / 2} G_{M} G_{Q}, \\
t_{22}=-\frac{1}{2 \sqrt{3} S} \eta G_{M}^{2}, \quad \text { with } \eta=Q^{2} / 4 M_{d}^{2}
\end{gathered}
$$

The tensor moment $t_{20}$ is particularly interesting due to its sensitivity to $G_{C}$. It has been previously measured using either the polarimeter or polarized target technique, up to $0.85(\mathrm{GeV} / c)^{2}$. In our experiment described below, new measurements of $t_{2 q}$ were performed between 0.66 and $1.7(\mathrm{GeV} / c)^{2} . A\left(Q^{2}\right)$ was measured previously up to $4(\mathrm{GeV} / c)^{2}$, but with significant discrepancy between data sets in our $Q^{2}$ range [8-10]. New $A\left(Q^{2}\right)$ data $[1,11]$, including some from this experiment, resolve many of these discrepancies. $B\left(Q^{2}\right)$, which is typically a factor of 10 smaller than $A\left(Q^{2}\right)$, has been measured up to $2.8(\mathrm{GeV} / c)^{2}$ [12].

Our experiment was performed at the Thomas Jefferson National Accelerator Facility (JLab) in the experimental Hall C (Fig. 1 shows a layout of the experimental setup). A continuous electron beam with a typical current between 80 and $120 \mu \mathrm{A}$ was used together with a $12 \mathrm{~cm}$ long liquid deuterium target resulting in an average luminosity of about $3 \times 10^{38} \mathrm{~cm}^{-2} \mathrm{~s}^{-1}$.

The scattered electrons were detected in the High Momentum Spectrometer (HMS), in coincidence with the recoil deuterons. The scattered deuterons were transported by a specially designed magnetic channel composed of warm magnets, three quadrupoles and one dipole, to the POLDER polarimeter. This magnetic channel optimized the acceptance matching between the two arms, which varied from 0.5 to 1 depending on the kinematics, and focused the elastically scattered deuterons on the target of POLDER. The deuteron magnetic channel was set at a

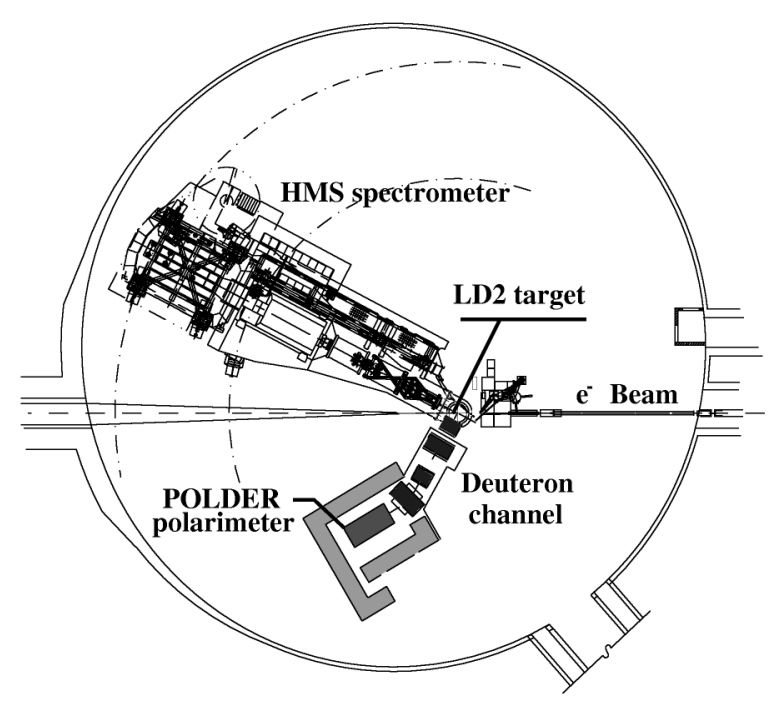

FIG. 1. Experimental setup of the $t_{20}$ experiment in Hall C at TJNAF. fixed angle of $60.5^{\circ}$. The six different $Q^{2}$ values were then obtained by changing both the beam energy (from 1.4 to $4 \mathrm{GeV}$ ) and the detection angle of the HMS spectrometer.

The elastic scattering events were selected by setting cuts on the primary vertex position and $\gamma^{*}-d$ invariant mass, as determined by the HMS, the particle energy loss in two thin plastic scintillators located before the polarimeter target, and the time coincidence measurement between the two arms. The combination of these redundant selection criteria reduced the contribution of remaining background (mainly coming from random coincidences between electrons and protons, and from coherent pion production) to less than $0.2 \%$.

The polarimeter POLDER $[13,14]$ is based on the charge exchange reaction ${ }^{1} \mathrm{H}(\vec{d}, 2 p) n$, which provides sizeable angular asymmetries depending on the tensor, but not on the vector, components of the incident deuteron polarization [15]. The direction of deuterons is measured with two multiwire proportional chambers placed upstream of a $22 \mathrm{~cm}$ long liquid hydrogen target. Deuterons that undergo a charge exchange reaction produce two outgoing protons with small relative angle and momentum in the forward direction. They are detected, and their positions measured, in two hodoscopes, composed of plastic scintillator bars. The polar and azimuthal angle distributions of the center of mass of the two protons are used to determine the deuteron beam polarization. The efficiency of the polarimeter, defined as the fraction of the deuterons undergoing a charge exchange reaction, is of the order of (3-6) $\times 10^{-3}$ and must be measured with a precision of $1 \%$. The absolute polarized efficiency $\epsilon_{\mathrm{pol}}(\theta, \varphi)$ of the polarimeter, measured in this experiment, has to be compared to the unpolarized value $\epsilon_{0}(\theta)$ through the relation:

$$
\begin{gathered}
\epsilon_{\mathrm{pol}}(\theta, \varphi)=\epsilon_{0}(\theta)\left[1+t_{20} T_{20}(\theta)+2 \cos (\phi) t_{21} T_{21}(\theta)\right. \\
\left.+2 \cos (2 \phi) t_{22} T_{22}(\theta)\right],
\end{gathered}
$$

where $T_{k q}$ are the analyzing powers of the ${ }^{1} \mathrm{H}(\vec{d}, 2 p) n$ reaction, $t_{k q}$ the deuteron polarization coefficients to be determined in this experiment, $\theta$ is the angle between the incident deuteron and the proton pair momentum, and $\phi$ the angle between the normal to the ${ }^{1} \mathrm{H}(\vec{d}, 2 p) n$ reaction plane and the $e-d$ scattering plane.

The analyzing powers and the unpolarized efficiency were measured previously at SATURNE using deuteron beams of known polarization in the range of kinetic energies between 140 and $520 \mathrm{MeV}$, in 10 to $30 \mathrm{MeV}$ steps [14]. The polarimeter data analysis was identical for the calibration and the JLab measurements. The selection of charge exchange events was achieved by requiring a coincidence between the detection of one incident particle before the target and the detection of two charged particles in the hodoscopes. Events with several incident particles were rejected using cuts on the energy loss measured in the scintillators and on the multiplicity 
information from the wire chambers. Time of flight was measured between the incident deuteron and the hit bars of hodoscopes. Cuts on this time of flight, together with an algorithm to reconstruct proper proton tracks, led to a clean selection of charge exchange events. Two different tracking algorithms, with different geometrical selection criteria, were used to prove that the background (parasitic reactions in the polarimeter or multiple incident particles not rejected by the front end of the polarimeter) within the charge exchange events was negligible. The angles $\theta$ and $\varphi$ were then calculated using the direction of the deuteron and the proton tracks. The deduced efficiency was then stable within $0.6 \%$ under changes of experimental conditions (except for the data at the lowest deuteron energy where variations reached $1.2 \%$ ).

The distributions of incident deuteron energy on the polarimeter at JLab had a large width, 16 to $51 \mathrm{MeV}$, and were not centered at any of the energies of the calibration experiment. The observables $\epsilon_{0}$ and $T_{k q}$ of Eq. (4) were then obtained by weighting with deuteron energies the interpolated SATURNE data. For this procedure, the deuteron energy was calculated for each event from the JLab beam energy and the scattered electron angle, with a correction coming from energy loss (mostly in the $\mathrm{LD}_{2}$ target).

The tensor polarization observables were obtained from Eq. (4) through a minimization procedure, adjusting the $t_{2 q}$ values such that the angular distribution on the righthand side best reproduced the angular distribution of the polarized efficiency measured in this experiment. In this fit, the resulting value of $t_{20}$ is highly correlated with the fixed value of $\epsilon_{0}$, but is uncorrelated with $t_{21}$ and $t_{22}$. A small spin precession correction was then applied, corresponding to a net deviation of $29.7^{\circ}$ in the deuteron channel. Our results [14,16-18] are given in Table I. The systematic errors include those due to analysis cuts (mostly from geometrical POLDER cuts), the uncertainties in the deuteron energy (from beam energy, electron angle, beam position on target), the uncertainties in calibration results (statistical and systematic errors on analyzing powers, interpolation, absolute stability on unpolarized efficiency), as well as the small instrumental unphysical asymmetries measured in the calibration. The uncertainty coming from the knowledge of the deuteron energy as well as the one due to calibration results was larger at the lowest $Q^{2}$ points because of the energy dependence of $\epsilon_{0}$ and the stability of the polarimeter at these deuteron energies. In the case of the point at $1.47(\mathrm{GeV} / c)^{2}$, the $\theta$ distribution of $\epsilon_{\mathrm{pol}}$ did not match exactly the expected behavior from Eq. (4). This led to the addition of a contribution to the systematic error in $t_{20}$ for this point of $\Delta t_{20}=0.1$. These systematic errors were combined quadratically and are mostly uncorrelated for the different data points.

For the sake of comparison with other data and with theoretical models, small corrections [of order $B / A$ and $B \tan ^{2}\left(\theta_{e} / 2\right) / A$; see Eqs. (1)-(3)] were applied to calculate $t_{2 q}$ at the conventionally accepted angle of $70^{\circ}$. These results obtained for the tensor polarization observables are shown in Figs. 2 and 3, and compared with the existing world data [2-7] and with several recent theoretical predictions. The error bars include both statistical and systematic errors, combined quadratically.

Where the new data overlap with the earlier Bates data [7], they agree within the combined uncertainties, although it appears that the Bates $t_{20}$ values are systematically more negative. The indication of $t_{21}$ crossing 0 is consistent with the existence of a node of the magnetic form factor $G_{M}$ [see Eq. (2)] around $2(\mathrm{GeV} / c)^{2}$, as first indicated by a measurement of $B\left(Q^{2}\right)$ [12].

A recent nonrelativistic impulse approximation prediction (NRIA) [19] calculated using the Argonne $v_{18}$ potential for the $N N$ interaction seems to reproduce the Bates data (the dotted curve in Figs. 2 and 3). But to be in a reasonable agreement with our new $t_{20}$ data, meson exchange currents (MEC) and relativistic corrections (RC) (solid curve) must be included. The MEC calculation includes pair terms and the $\rho \pi \gamma$ mechanism, for which the strength is not well known [20].

Two relativistic and covariant models, both including MEC, are compared with the data. The dashed curve [21] uses a three-dimensional reduction of the Bethe-Salpeter equation using an equal-time formalism, and includes $\rho \pi \gamma$ exchange currents. The long dashed curve [22] is the prediction of a model developed in the framework of the explicitly covariant version of light front dynamics. It uses a full relativistic potential, calculated with the

TABLE I. Measured tensor Polarization observables $t_{k q}\left(\theta_{e}\right)$, with statistical and systematic errors. The charge form factors are given with, in some occurrences, asymmetric overall errors.

\begin{tabular}{|c|c|c|c|c|c|c|}
\hline$Q^{2}(\mathrm{GeV} / c)^{2}$ & 0.651 & 0.775 & 1.009 & 1.165 & 1.473 & 1.717 \\
\hline$\theta_{e}(\mathrm{deg})$ & 35.6 & 33.4 & 29.8 & 27.3 & 23.0 & 19.8 \\
\hline $\begin{aligned} & \pm \Delta \text { stat } \\
t_{20} & \pm \Delta \text { syst }\end{aligned}$ & $-0.546_{ \pm 0.170}^{ \pm 0.038}$ & $-0.322 \stackrel{ \pm 0.031}{ \pm 0.088}$ & $0.191_{ \pm 0.043}^{ \pm 0.034}$ & $0.301 \stackrel{ \pm 0.048}{ \pm 0.056}$ & $0.625_{ \pm 0.141}^{ \pm 0.094}$ & $0.477 \stackrel{ \pm 0.178}{ \pm 0.063}$ \\
\hline $\begin{array}{r}t_{21} \begin{array}{l} \pm \text { stat } \\
\pm \Delta \text { syst }\end{array}\end{array}$ & $0.463_{ \pm 0.113}^{ \pm 0.051}$ & $0.315_{ \pm 0.083}^{ \pm 0.041}$ & $0.201 \stackrel{ \pm 0.042}{ \pm 0.077}$ & $0.220_{ \pm 0.094}^{ \pm 0.056}$ & $0.166_{ \pm 0.056}^{ \pm 0.096}$ & $-0.001 \stackrel{ \pm 0.152}{ \pm 0.058}$ \\
\hline $\begin{array}{r}t_{22} \pm \Delta \text { stat } \\
\pm \Delta \text { syst }\end{array}$ & $0.087 \stackrel{ \pm 0.042}{ \pm 0.037}$ & $-0.027 \stackrel{ \pm 0.030}{ \pm 0.037}$ & $-0.018_{ \pm 0.029}^{ \pm 0.029}$ & $0.022 \stackrel{ \pm 0.035}{ \pm 0.037}$ & $-0.023_{ \pm 0.048}^{ \pm 0.054}$ & $-0.133_{ \pm 0.047}^{ \pm 0.074}$ \\
\hline$G_{C} \times 10^{2}$ & $-0.117 \pm 0.162$ & $-0.253 \pm 0.063$ & $-0.396 \pm 0.028$ & $-0.348 \pm 0.031$ & $-0.310_{-0.061}^{+0.053}$ & $-0.194_{-0.052}^{+0.036}$ \\
\hline$G_{Q}$ & $0.393 \pm 0.010$ & $0.259 \pm 0.007$ & $0.122 \pm 0.004$ & $0.080 \pm 0.003$ & $0.034_{-0.007}^{+0.005}$ & $0.023_{-0.004}^{+0.002}$ \\
\hline
\end{tabular}




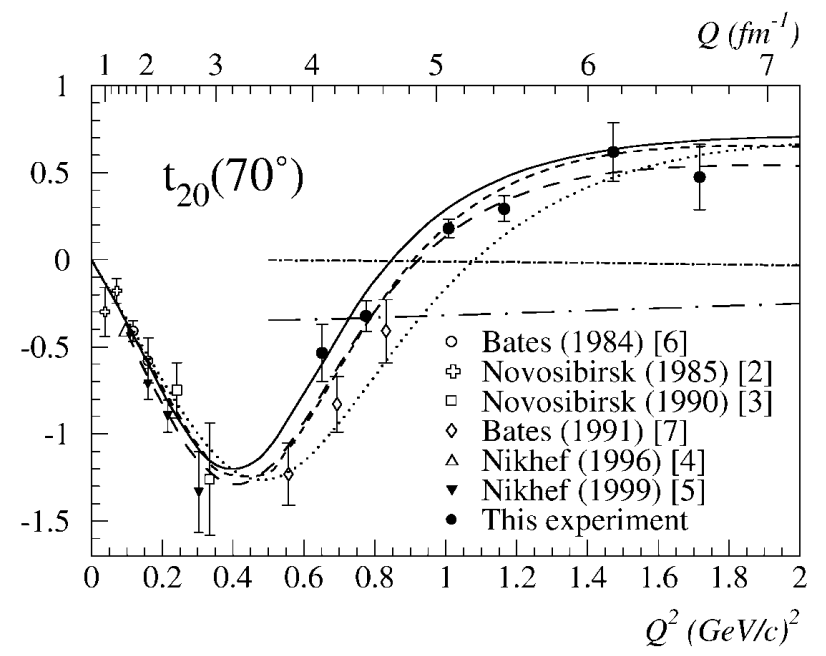

FIG. 2. $t_{20}$ at $\theta_{e}=70^{\circ}$ compared to theoretical predictions; dotted line (NRIA) and full line (NRIA + MEC + RC) [19]; relativistic models with dashed line [21] and long dashed line [22]; pQCD calculations with dash-dotted line [23] and long dash-dotted line [24].

same set of mesons and parameter values used in the construction of the Bonn potential, but does not include the $\rho \pi \gamma$ MEC. Both models are in good agreement with our $t_{20}$ data, but the prediction based on the light cone formalism agrees better with the last NIKHEF data, at lower $Q^{2}$ [5]. However, this model does not reproduce the position of the node of $G_{M}$, which leads to a bad description of $t_{21}$.

Finally, two perturbative QCD (pQCD) calculations, predicting simple relations between the form factors of the deuteron, are shown by dash-dotted curves in Figs. 2 and 3. One of them [23] uses only the helicity-conserving matrix element of the electromagnetic current, arguing that it should dominate above $1(\mathrm{GeV} / c)^{2}$. The other one [24] includes the helicity-one-flip matrix element and fixes its contribution using the location of the node of $B\left(Q^{2}\right)$, taken to be at $2(\mathrm{GeV} / c)^{2}$. Comparison with $t_{20}$ and $t_{21}$ measurements clearly shows that both $\mathrm{pQCD}$ predictions fail to reproduce our data contrary to the scale in four-

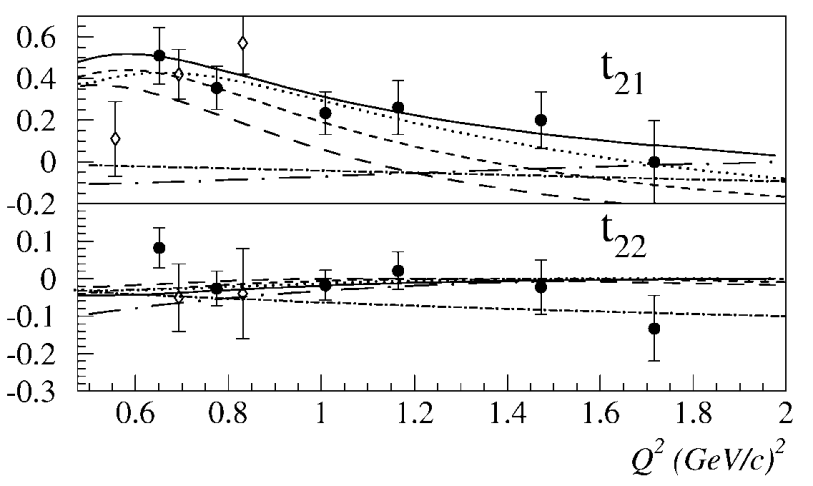

FIG. 3. $t_{21}$ and $t_{22}$ at $\theta_{e}=70^{\circ}$. See Fig. 2 and text for the curves. momentum transfer given by the authors for the applicability of their calculations.

Deuteron form factors can be expressed in terms of $A$ (which have been interpolated using the latest data $[1,11]$ in our $Q^{2}$ range), $B$, and $t_{20}$. These equations are quadratic and admit, in general, two solutions. Ambiguities in the choice of the proper solution remain only for our two highest $Q^{2}$ points, due to the fact that $t_{20}$ is close to its maximum, where the two solutions are nearly degenerate. If we follow the prediction of most theoretical models, according to which the maximum of $t_{20}$ occurs beyond our highest $Q^{2}$ point, one of the two solutions can be selected. This particular issue will be addressed elsewhere in more detail [25]. The errors in $G_{C}$ (see Table I) come predominantly from the errors in the $t_{20}$ measurements.

The results for the charge form factors $G_{C}$ and $G_{Q}$, shown in Fig. 4, lead to the same conclusions made for $t_{20}$ data about the models and the Bates data. The results for the charge form factor $G_{C}$ show a node located at a lower value than inferred from the previous Bates data. This removes the inconsistency, pointed out by Henning [26], in the location of the minimum for the charge form factor of two- and three-nucleon systems. Our data also suggest for the first time a secondary maximum of $\left|G_{C}\right|$. The height of this maximum seems to be inconsistent with that of the corresponding three-nucleon system, within the same nonrelativistic models [26].

In summary, we have measured the tensor polarization observables in electron-deuteron elastic scattering between 0.65 and $1.72(\mathrm{GeV} / c)^{2}$. Our data on $t_{20}$, used in conjunction with data on the structure function $A\left(Q^{2}\right)$, provide a determination of the charge and quadrupole form factors. We have compared our results with only a few recent calculations. Within nonrelativistic models, all the observables are in favor of the inclusion of meson exchange currents and relativistic effects in the theoretical calculations. In fact, the present data could constitute the best experimental determination of isoscalar meson exchange currents. Recent relativistic models are in remarkable agreement with our data. Finally, the $Q^{2}$ range covered by these data shows
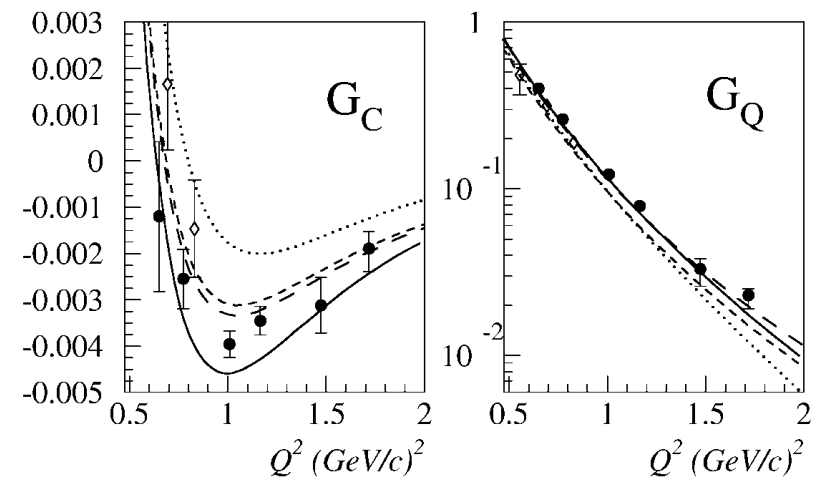

FIG. 4. Monopole $\left(G_{C}\right)$ and quadrupole $\left(G_{Q}\right)$ charge form factors of the deuteron. See Fig. 2 and text for the curves and references. 
that the $\mathrm{pQCD}$ predictions are not reliable for these momentum transfers.

We acknowledge the outstanding work of the JLab accelerator division and the Hall $\mathrm{C}$ engineering staff. We thank the Indiana University Cyclotron Facility for its technical help. This work was supported by the French Centre National de la Recherche Scientifique and Commissariat à l'Energie Atomique, the U.S. Department of Energy and National Science Foundation, the Swiss National Science Foundation, and the K. C. Wong Foundation.

[1] D. Abbott et al., Phys. Rev. Lett. 82, 1379 (1999).

[2] V.F. Dmitriev et al., Phys. Lett. 157B, 143 (1985).

[3] R. Gilman et al., Phys. Rev. Lett. 65, 1733 (1990).

[4] M. Ferro-Luzzi et al., Phys. Rev. Lett. 77, 2630 (1996).

[5] M. Bouwhuis et al., Phys. Rev. Lett. 82, 3755 (1999).

[6] M.E. Schulze et al., Phys. Rev. Lett. 52, 597 (1984).

[7] M. Garçon et al., Phys. Rev. C 49, 2516 (1994).

[8] R. Cramer et al., Z. Phys. C 29, 513 (1985).

[9] J.E. Elias et al., Phys. Rev. 177, 2075 (1969).

[10] R. G. Arnold et al., Phys. Rev. Lett. 35, 776 (1975).

[11] L. C. Alexa et al., Phys. Rev. Lett. 82, 1374 (1999).

[12] P. Bosted et al., Phys. Rev. C 42, 38 (1990), and references therein.
[13] S. Kox et al., Nucl. Instrum. Methods Phys. Res., Sect. A 346, 527 (1994); J.-S. Real, Ph.D. thesis, University of Grenoble 1 (Inst. Report No. ISN-94-04, 1994).

[14] L. Eyraud, Ph.D. thesis, University of Grenoble 1 (Inst. Report No. ISN-98-101, 1998).

[15] D. V. Bugg and C. Wilkin Nucl. Phys. A467, 575 (1987); J. Carbonell, M. B. Barbaro, and C. Wilkin, Nucl. Phys. A529, 653 (1991).

[16] K. Gustafsson, Ph.D. thesis, University of Maryland (unpublished).

[17] K. Hafidi, Ph.D. thesis, University of Paris XI-Orsay (Inst. Report No. DAPNIA/SPhN-99-05T, 1999).

[18] W. Zhao, Ph.D. thesis, MIT, 1999 (unpublished).

[19] R. B. Wiringa, V. G. J. Stoks, and R. Schiavilla, Phys. Rev. C 51, 38 (1995).

[20] J. W. Van Orden, N. Devine, and F. Gross, Phys. Rev. Lett. 75, 4369 (1995).

[21] D. R. Phillips, S. J. Wallace, and N. K. Devine, nucl-th/ 9906086.

[22] J. Carbonell and V.A. Karmanov, Eur. Phys. J. A 6, 9 (1999).

[23] S. J. Brodsky and J. R. Hiller, Phys. Rev. D 46, 2141 (1992).

[24] A. Kobushkin and A. Syamtomov, Phys. Rev. D 49, 1637 (1994).

[25] D. Abbott et al., Eur. Phys. J. A 7, 421 (2000).

[26] H. Henning et al., Phys. Rev. C 52, 471 (1995). 